\title{
Reconnections of Vortex Loops in the Superfluid Turbulent HeII. Rates of the Breakdown and Fusion processes.
}

\author{
Sergey K. Nemirovskii \\ Institute for Thermophysics, Lavrentyeva, 1, 630090 Novosibirsk, Russia
}

(Dated: today)

\begin{abstract}
Kinetics of merging and breaking down vortex loops is the important part of the whole vortex tangle dynamics. Another part is the motion of individual lines, which obeys the Biot-Savart law in presence of friction force and of applied external velocity fields if any. In the present work we evaluate the coefficients of the reconnection rates $A\left(l_{1}, l_{2}, l\right)$ and $B\left(l, l_{1}, l_{2}\right)$. Quantity $A$ is a number (per unit of time and per unit of volume) of events, when two loops with lengths $l_{1}$ and $l_{2}$ collide and form the single loop of length $l=l_{1}+l_{2}$. Quantity $B\left(l, l_{1}, l_{2}\right)$ describes the rate of events, when the single loop of the length $l$ breaks down into two the daughter loops of lengths $l_{1}$ and $l_{2}$. These quantities ave evaluated as the averaged numbers of zeroes of vector $\mathbf{S}_{s}\left(\xi_{2}, \xi_{1}, t\right)$ connecting two points on the loops of $\xi_{2}$ and $\xi_{1}$ at moment of time $t$. Statistics of the individual loops is taken from the Gaussian model of vortex tangle.
\end{abstract}

PACS-number 67.40

\section{INTRODUCTION}

Quantized vortices appeared in quantum fluids and other systems play a fundamental role in the properties of the latter. For that reason they have been an object of intensive study for many years (for review and bibliography see e. g. [1]). The greatest success in investigations of dynamics of quantized vortices has been achieved in relatively simple cases such as a vortex array in rotating helium or vortex rings. However these simple cases are rather exception than a rule. Due to extremely involved dynamics initially straight lines or rings evolve to form highly chaotic structure, so called vortex tangle (see e.g. papers [2], [3] and review [4]). The vortex tangle consists of a set of vortex loops of different lengths. The individual vortex loops evolves obeying the Biot-Savart law, undergoing the friction force and the applied external velocity field. All these processes are good understood, although the according equations of motion are highly nonlinear (and nonlocal) therefore general analysis can be made with use of numerical methods.

Besides motion of each of the individual loops there is one more element of general evolution of the vortex tangle related to collision of loops, or intersection of elements of vortex lines. During intersection of lines the very complicated process, related to arrangement of the vortex core takes place [5]. Nevertheless this process is relatively short, therefore it is usually accepted that the filaments instantly reconnect whenever they intersect each other. It is widely appreciated that the reconnection processes influence both the structure and evolution of vortex tangle. However the questions how it happens and what mechanisms are responsible for these remain open. For instance, Feynman in his pioneering paper devoted to superfluid turbulence proposed scenario how the vortex tangle decays in absence of applied external counterflow. According to this scenario a fusion of small vortex rings into larger ones as well as a breakdown into smaller ones is possible at the moments of the reconnection events (see Fig. 1). In assumption that on the average

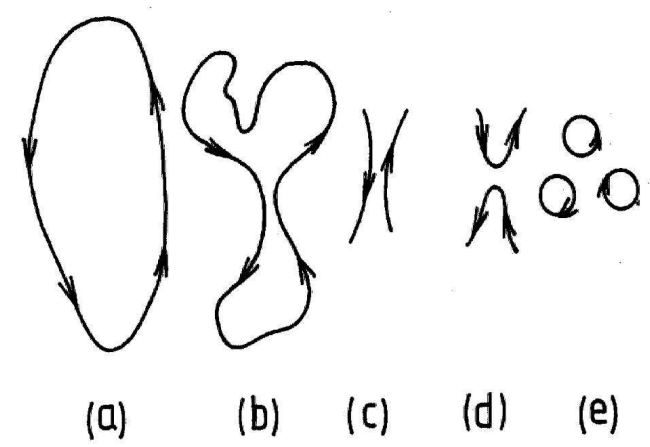

FIG. 1: The reconnection process schematically (Feynman, 1955, Fig. 10). (a) initial stage, (b) and (c) are stages of collapse, (d) reconnection stage, (e) stage of degeneration of vortex rings into thermal excitations. 


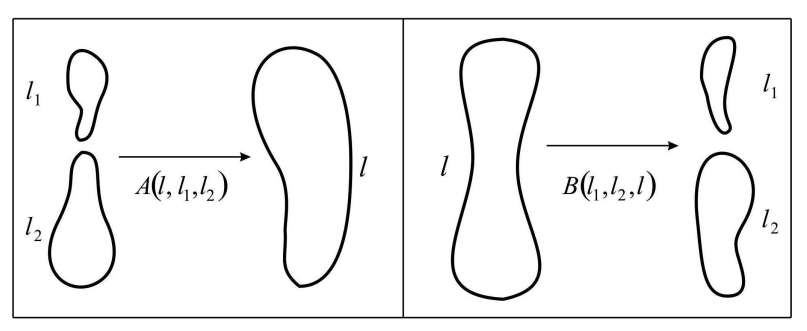

FIG. 2: Schematic sketch of the fusion and breaking-down of vortex loops. Rates of these processes characterized by the coefficients $A\left(l_{1}, l_{2}, l\right)$ and $B\left(l, l_{1}, l_{2}\right)$.

the last property dominates i.e. the cascade like process of formation of smaller and smaller loops forms. When the scale of the small rings becomes of the order of the interatomic distances, which is the final stage of the cascade, the vortex motion is degenerated into thermal excitations. This key idea that degeneration of the vortex tangle occurs due to cascade-like transferring of the length in space of scale of sizes of vortex loops was indirectly confirmed only in numerical calculations, where the procedure of artificial elimination of small loops had been used [6]-[9].

In spite of the recognized importance of the reconnecting loops kinetics, the numerical results remain main source of information about this process. The obvious lack of theoretical investigations interferes with deep insight in the nature of this phenomena (this question had been recently discussed in [10]). For instance it is not clear how the cascade of length in space of vortex loops sizes is formed, what mechanisms are responsible for this, what quantities determine an intensity of cascade, and why at all breakdown of the loop prevails.

Of course the scarcity of analytic investigations related to incredible complexity of the problem. Indeed we have to deal the set of objects with not fixed number of elements, they can born and die. Thus, some analog of the secondary quantization method is required with the difference that objects (vortex loops) themselves possess an infinite number of degree of freedom with very involved dynamics. Clearly this problem can hardly be resolved in nearest future. Recently in [11] much more modest approach, based on the "rate equation" for distribution function $n(l)$ was elaborated in context of cosmic strings. Following this work we introduce distribution function $n(l, t)$ of density of loop in "space" of their lengths. It is defined as a number of loops (per unit of volume) with lengths between $l$ and $l+d l$. Due to reconnection processes $n(l, t)$ can vary.

We discriminate two types of processes, namely the fusion of two loops into the larger single loop and the breakdown of single loop into two daughter loops. The kinetic of vortex tangle is affected by the intensity of the introduced processes. The intensity of the first process is characterized by the rate of collision $A\left(l_{1}, l_{2}, l\right)$ of two loops with lengths $l_{1}$ and $l_{2}$ and forming the loop of length $l=l_{1}+l_{2}$. The intensity of the second process is characterized by the rate of self-intersection $B\left(l, l_{1}, l_{2}\right)$ of loop of the length $l$ into two daughter loops with lengths $l_{1}$ and $l_{2}$ (see Fig. 2). In view of exposed above we can directly write out the master "kinetic" equation for rate of change distribution function $n(l, t)$.

$$
\begin{array}{rlrl}
\iint A\left(l_{1}, l_{2}, l\right) n\left(l_{1}\right) n\left(l_{2}\right) \delta\left(l-l_{1}-l_{2}\right) d l_{1} d l_{2} & \frac{\partial n(l, t)}{\partial t} & = \\
l_{1}+l_{2} & \rightarrow l \\
-\iint A\left(l_{1}, l, l_{2},\right) \delta\left(l_{2}-l_{1}-l\right) n(l) n\left(l_{1}\right) d l_{1} d l_{2} & l_{1}+l & \rightarrow l_{2} \\
-\iint A\left(l_{2}, l, l_{1},\right) \delta\left(l_{1}-l_{2}-l\right) n(l) n\left(l_{1}\right) d l_{1} d l_{2} & l_{2}+l & \rightarrow l_{1} \\
-\iint B\left(l_{1}, l_{2}, l\right) n(l) \delta\left(l-l_{1}-l_{2}\right) d l_{1} d l_{2} & l & \rightarrow l_{1}+l_{2} \\
+\iint B\left(l, l_{2}, l_{1}\right) \delta\left(l_{1}-l-l_{2}\right) n\left(l_{1}\right) d l_{1} d l_{2} & l_{1} & \rightarrow l+l_{2} \\
+\iint B\left(l, l_{1}, l_{2}\right) \delta\left(l_{2}-l-l_{1}\right) n\left(l_{1}\right) d l_{1} d l_{2} & l_{2} & \rightarrow l+l_{1}
\end{array}
$$

All of the processes are depicted at the left of each line. Clear that the "kinetic" equation has "bookkeeping" character. Physics of this approach lies in the "correct" determinations of coefficient of that equations $A\left(l_{1}, l_{2}, l\right)$ and $B\left(l, l_{1}, l_{2}\right)$ on the base of some more or less plausible model. 


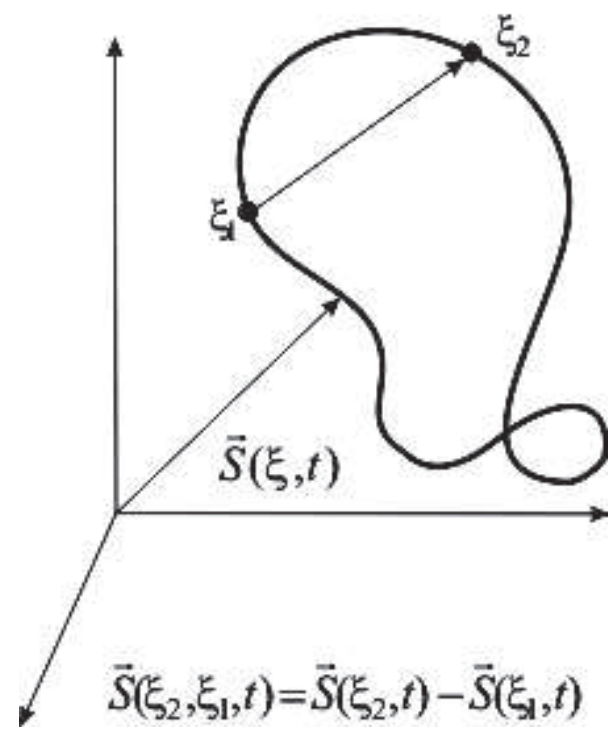

FIG. 3: Schematic sketch of vortex loop. Elements of line are described as vectors $\mathbf{s}(\xi)$, where the label variable $\xi$ is taken here as the arc length. We associate the moment of intersection with the vanishing of vector $\mathbf{S}\left(\xi_{1}, \xi_{1}, t\right)$ connecting points $\mathbf{s}\left(\xi_{2}, t\right)$ and $\mathbf{s}\left(\xi_{1}, t\right)$.

In the present work we derive some general (independent on the model) relations for reconnection rates $A\left(l_{1}, l_{2}, l\right)$ and $\left.B\left(l, l_{1}, l_{2}\right)\right)$ ( Section II). Then in Sec. III we apply these relations to evaluate the reconnection rates on the base of Gaussian model of the vortex tangle elaborated earlier [12]. Section IV is devoted to conclusion and plans for future investigations.

\section{MATHEMATICAL IDENTITIES FOR $A\left(l_{1}, l_{2}, l\right)$ AND $B\left(l, l_{1}, l_{2}\right)$.}

In this section we will formulate mathematical definition for quantities $A\left(l_{1}, l_{2}, l\right)$ and $B\left(l, l_{1}, l_{2}\right)$. We will start with the latter quantity $B\left(l, l_{1}, l_{2}\right)$. By definition its physical meaning is just frequency of events when part of line with total length $l$ intersects the same line to create two two daughter loops with lengths $l_{1}$ and $l_{2}$ (see Fig. 2), that is self-crossing event. As it is already stated we assume that each crossing event leads to reconnection of lines. The elements of vortex line are described as a function $\mathbf{s}(\xi, t)$ (See Fig. 3) that is time dependant radius-vector of the points resting on loop. Variable $\xi$ labels the points of the loop. It is convenient to choose variable $\xi$ to be equal to the arc length, $(0 \leq \xi \leq l)$. Let us consider function

$$
\mathbf{S}_{s}\left(\xi_{2}, \xi_{1}, t\right)=\mathbf{s}\left(\xi_{2}, t\right)-\mathbf{s}\left(\xi_{1}, t\right),
$$

that is just $3 D$ vector connecting points $\mathbf{s}\left(\xi_{2}, t_{2}\right)$ and $\mathbf{s}\left(\xi_{1}, t_{1}\right)$. Clearly that condition $\mathbf{S}_{b}\left(\xi_{2}, \xi_{1}, t\right)=0$ implies that the self-crossing event of parts of line with label-coordinates $\xi_{2}, \xi_{1}$ occurs at moment of time $t$. The quantity $\mathbf{S}_{b}\left(\xi_{2}, \xi_{1}, t\right)$ is fluctuating 3-component function of three arguments $\xi_{2}, \xi_{1}, t$. We are interested in how often $\mathbf{S}_{b}\left(\xi_{2}, \xi_{1}, t\right)$ vanishes in cube of space $\zeta=\left\{\xi_{2}, \xi_{1}, t\right\}$. Points in space $\zeta=\left\{\xi_{2}, \xi_{1}, t\right\}$ where function $\mathbf{S}_{b}\left(\xi_{2}, \xi_{1}, t\right)$ vanishes, are points of cross of 3 surfaces $S_{i}\left(\xi_{2}, \xi_{1}, t\right)=0, i=x, y, z$ in space $\zeta=\left\{\xi_{2}, \xi_{1}, t\right\}$ as it is shown in Fig. 4 .

From theory of generalized function it follows that number of these points (we denote them below as $\zeta_{a}$ ) can be expressed via $\delta$-function of quantity $\mathbf{S}_{b}\left(\xi_{2}, \xi_{1}, t\right)$ with the help of following formula .

$$
\sum_{a} \delta\left(\zeta-\zeta_{a}\right)=\left|\frac{\partial(X, Y, Z)}{\partial\left(\xi_{2}, \xi_{1}, t\right)}\right|_{\zeta=\zeta_{a}} \delta\left(\mathbf{S}_{b}\left(\xi_{2}, \xi_{1}, t\right)\right)
$$

Here $X, Y, Z$ are the components of vector $\mathbf{S}_{b}\left(\xi_{2}, t_{2}, \xi_{1}, 0\right)$. By integration of both parts of (3) over $d \xi_{1} d \xi_{2}$ we would obtain the full number of intersections (per unit time). If, further to introduces additional constraint $\delta\left(\xi_{2}-\xi_{1}-l_{1}\right)$ implying that the distance (along line) between chosen points is equal to $l$ and to integrate over $d \xi_{1} d \xi_{2}$, we obtain the rate of self-intersection of line with length $l$ and breakdown it into pieces $l_{1}$ and $l-l_{1}$. 


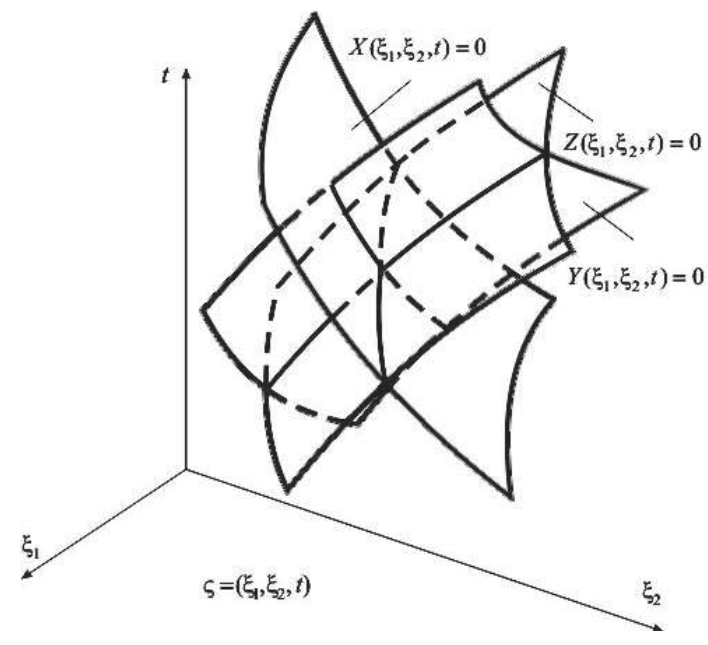

FIG. 4: The reconnection events can be described as zeroes of function $\mathbf{S}_{b}\left(\xi_{2}, \xi_{1}, t\right)$. In space of its variables $\zeta=\left\{\xi_{2}, \xi_{1}, t\right\}$ these points are cross of 3 surfaces $S_{i}\left(\xi_{2}, \xi_{1}, t\right)=0, \quad i=x, y, z$.

In addition we have to do averaging over all possible fluctuating configurations. Thus the coefficient $B\left(l_{1}, l-l_{1}, l\right)$ with dimension $[s]=s^{-1} \mathrm{~cm}^{-1}$ is equal to

$$
B\left(l_{1}, l-l_{1}, l\right)=\iint d \xi_{1} d \xi_{2} \delta\left(\xi_{2}-\xi_{1}-l_{1}\right)\left\langle\left|\frac{\partial(X, Y, Z)}{\partial\left(\xi_{2}, \xi_{1}, t\right)}\right|_{\zeta=\zeta_{a}} \delta\left(\mathbf{S}_{b}\left(\xi_{2}, \xi_{1}, t\right)\right)\right\rangle .
$$

To obtain coefficient $A\left(l_{1}, l_{2}, l\right)$ we use the similar procedure. Let us consider two loops with length $l_{1}$ and $l_{2}$. Our purpose now to find the rate $A\left(l_{1}, l_{2}, l\right)$ of fusion of these two loops into one loop of length $l=l_{1}+l_{2}$. Dimension of $A\left(l_{1}, l_{2}, l\right)$ is $[A]=\mathrm{cm}^{3} \mathrm{~s}^{-1}$. As previously we describe vortex filaments by positions of radius vectors their elements $\mathbf{s}\left(\xi_{1}, t\right)$ and $\mathbf{s}\left(\xi_{2}, t\right)$. Here we have two label variables $\xi_{1}, \xi_{2}$ belonging to different loops and running in limits $(0 \leq \xi \leq$ $\left.l_{1}\right)$ and $\left(0 \leq \xi \leq l_{2}\right)$ respectively. One more important difference with the previous case is that both functions $\mathbf{s}\left(\xi_{1}, t\right)$ and $\mathbf{s}\left(\xi_{2}, t\right)$ should depend on "initial" positions $\mathbf{s}\left(\xi_{2}=0, t\right)=\mathbf{R}_{1}(t)$ and $\mathbf{s}\left(\xi_{2}=0, t\right)=\mathbf{R}_{2}(t)$, chosen arbitrary. Of course in previous case of self-intersection of single loop, quantity $\mathbf{s}(\xi, t)$ also depended on "initial" positions $\mathbf{R}(\mathbf{t})$, but it did not influence the rate of self-intersection. Now for case of the fusion this dependance is important, since very distant loops have small probability to collide. Let us introduce the "fusion" functions

$$
\mathbf{S}_{f}\left(\xi_{2}, \xi_{1}, t_{1}\right)=\mathbf{s}\left(\xi_{2}, t\right)-\mathbf{s}\left(\xi_{1}, t\right) .
$$

Repeating the considerations for case of the single loop we find that number of reconnection (per unit of time) of points $\xi_{2}, \xi_{1}$ formally coincides with (3)

$$
\sum \delta\left(\zeta-\zeta_{a}\right)=\left|\frac{\partial(X, Y, Z)}{\partial\left(\xi_{2}, \xi_{1}, t\right)}\right|_{\zeta=\zeta_{a}} \delta\left(\mathbf{S}_{f}\left(\xi_{2}, \xi_{1}, t\right)\right)
$$

with the difference that $\xi_{2}, \xi_{1}$ belong to different curves. Since intersections of any elements of lines lead to fusion of the loops we have to integrate (6) over $d \xi_{1} d \xi_{2}$. The result obtained is valid for chosen pair of loops. To obtain the total number of events we have to multiply the result obtained by quantity $n\left(l_{1}\right) n\left(l_{2}\right) d \mathbf{R}_{1} d \mathbf{R}_{2}$, which is just a full number of loops of chosen sizes in the whole volume. Comparing with the master kinetic equation (1) we find the final expression for fusion coefficient $A\left(l_{1}, l_{2}, l\right)$

$$
A\left(l_{1}, l_{2}, l\right)=\frac{1}{\mathrm{VOLUME}} \iint d \mathbf{R}_{1} d \mathbf{R}_{2} \iint d \xi_{1} d \xi_{2}\left\langle\left|\frac{\partial(X, Y, Z)}{\partial\left(\xi_{2}, \xi_{1}, t\right)}\right|_{\zeta=\zeta_{a}} \delta\left(\mathbf{S}\left(\xi_{2}, \xi_{1}, t\right)\right)\right\rangle .
$$

Thus we obtained expressions (4)and (7), which allow to calculate the rates of reconnections of the fusion and breakdown vortex loops. They are however just formal mathematical identities. Concrete results depend on statistics of individual lines. Therefore to move further we have to ascertain the procedure for averaging. It will be done in the next section. 


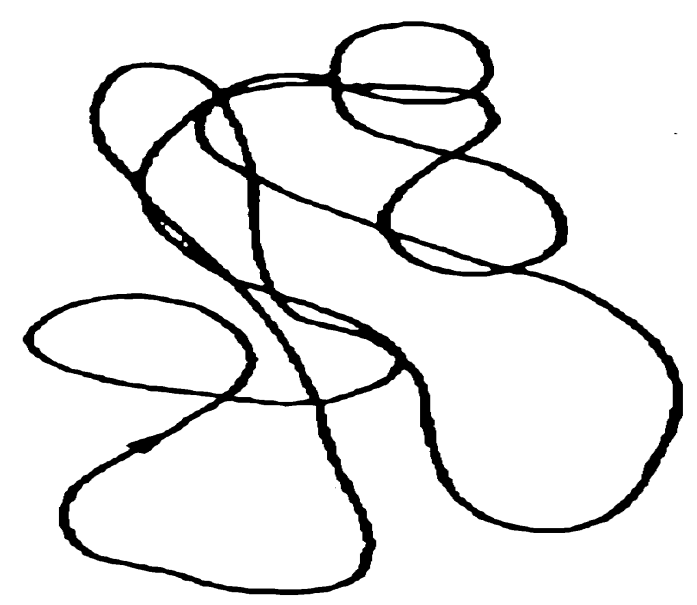

FIG. 5: Snapshot of the "average" vortex loop obtained from analysis of the statistical properties. Close $(\Delta \xi \ll R)$ parts of the line are separated in $3 D$ space by distance $\Delta \xi$. The distant parts $(R \ll \Delta \xi)$ are separated in $3 D$ space by the distance $\sqrt{\xi_{0} \Delta \xi}$, i.e. the vortex loop has the typical random walking structure.

\section{GAUSSIAN MODEL CASE}

\section{A. The generalized Wiener distribution}

To evaluate quantities $B\left(l_{1}, l_{2}, l\right)$ and $A\left(l_{1}, l_{2}, l\right)$ written in form (4) and (7) one needs to know statistics of individual loops. In general case this statistics should be extracted from investigation of the full dynamical problem. The according statement of such problem includes equation of motion (Biot-Savart law for quantum vortices), dissipative effects (interaction with normal component) and additional Langevin force responsible for chaotic behavior. The problem becomes more involved by circumstance that other loops also influence dynamics of the chosen loop. At this stage we choose another way, namely we use the Gaussian model of the vortex tangle elaborated by author [12]. To develop this approach the trial distribution functional of Gaussian form had been consructed. This functional absorbed all properties of the superfluid turbulence known from both experimental studies and numerical simulations. According to this model the "average" vortex loop has a typical structure shown in Fig. 5. The close parts of the loop separated (along line) by distance $\xi_{2}-\xi_{1}$ smaller then the mean radius of curvature $\xi_{0}$ are strongly correlated, $\left\langle\mathbf{s}^{\prime}\left(\xi_{1}, t\right) \mathbf{s}^{\prime}\left(\xi_{2}, t\right)\right\rangle \rightarrow 1$, ( $\mathbf{s}^{\prime}$ is the tangent vector) and line is smooth. Remote parts of the line $\left(\xi_{2}-\xi_{1} \gg \xi_{0}\right)$ are not correlated at all, $\left\langle\mathbf{s}^{\prime}\left(\xi_{1}, t\right) \mathbf{s}^{\prime}\left(\xi_{2}, t\right)\right\rangle \rightarrow 0$. Thus for large separations the vortex loop has a typical "random walking structure" with the Wiener distribution. This "semifractal" behavior satisfies to the generalized Wiener distribution. Namely, the probability $\mathcal{P}(\{\mathbf{s}(\xi, t)\})$ to find some particular configuration $\{\mathbf{s}(\xi, t)\}$ is expressed by the probability distribution functional (see for details in [12])

$$
\mathcal{P}(\{\mathbf{s}(\xi, t)\})=\mathcal{N} \exp \left(-\int_{0}^{l} \int_{0}^{l} \mathbf{s}^{\prime \alpha}\left(\xi_{1}, t\right) \Lambda^{\alpha \beta}\left(\xi_{1}-\xi_{2}\right) \mathbf{s}^{\prime \beta}\left(\xi_{2}, t\right) d \xi_{1} d \xi_{2}\right) .
$$

Here $\mathcal{N}$ is normalizing factor, $l$ is the length of curve, it is supposed to be much larger then mean radius of curvature $\xi_{0}$. The probability distribution functional (8) introduced in [12] distribution considered anisotropy and polarization of vortex tangle in counterflowing turbulent helium. This fact is reflected by the circumstance that $\Lambda^{\alpha \beta}\left(\xi_{1}-\xi_{2}\right)$ is the full matrix. In practice it is more convenient to deal with characteristic functional $W(\{\mathbf{P}(\xi, t)\})$ defined as

$$
W(\{\mathbf{P}(\xi, t)\})=\left\langle\exp \left(i \int_{0}^{l} \mathbf{P}(\xi, t) \mathbf{s}^{\prime}(\xi, t) d \xi\right)\right\rangle .
$$

The characteristic functional enables us calculate any averages depending on vortex lines configuration $\{\mathbf{s}(\xi, t)\}$ by simple functional differentiation. For instance the average tangent vector $\left\langle\mathbf{s}_{\alpha}^{\prime}\left(\xi_{1}\right)\right\rangle$ or the correlation function between 
orientation of the different elements of the vortex filaments $\left\langle\mathbf{s}_{\alpha}^{\prime}\left(\xi_{1}\right) \mathbf{s}_{\beta}^{\prime}\left(\xi_{2}\right)\right\rangle$ are readily expressed via characteristic functional accordingly to the following rules:

$$
\left\langle\mathbf{s}_{\alpha}^{\prime}\left(\xi_{1}\right)\right\rangle=\left.\frac{\delta W}{i \delta \mathbf{P}^{\alpha}\left(\xi_{1}\right)}\right|_{\mathbf{P}=0}, \quad\left\langle\mathbf{s}_{\alpha}^{\prime}\left(\xi_{1}\right) \mathbf{s}_{\beta}^{\prime}\left(\xi_{2}\right)\right\rangle=\left.\frac{\delta^{2} W}{i \delta \mathbf{P}^{\alpha}\left(\xi_{1}\right) i \delta \mathbf{P}^{\beta}\left(\xi_{2}\right)}\right|_{\mathbf{P}=0}
$$

Calculation of the characteristic functional $W(\{\mathbf{P}(\xi, t)\})(9)$ on the base of the probability functional (8) is reduced to functional integration, which, in turn, reduces to the "full square procedure". The result is

$$
W(\{\mathbf{P}(\xi, t)\})=\exp \left\{-\int_{0}^{l} \int_{0}^{l} \mathbf{P}^{\alpha}\left(\xi_{1}\right) N^{\alpha \beta}\left(\xi_{1}-\xi_{2}\right) \mathbf{P}^{\beta}\left(\xi_{2}\right) d \xi_{1} d \xi_{2}\right\} .
$$

To avoid unnecessary lengthy calculations we simplify the model expressed by the probability distribution functional (8), namely we omit both the anisotropy and polarization. In this case the matrix $N^{\alpha \beta}\left(\xi_{1}-\xi_{2}\right)$ used in ([12]) can be taken as

$$
N^{\alpha \beta}\left(\xi_{1}-\xi_{2}\right)=\delta_{\alpha \beta} \frac{1}{6\left(1-2 \xi_{0} \sqrt{\pi} / l\right)}\left(\exp \left[-\frac{\left(\xi_{1}-\xi_{2}\right)^{2}}{4 \xi_{0}^{2}}\right]-\frac{2 \xi_{0} \sqrt{\pi}}{l}\right) .
$$

For small separation $\left(\xi_{2}-\xi_{1} \ll \xi_{0}\right), \sum_{\alpha} N^{\alpha}\left(\xi_{1}-\xi_{2}\right) \rightarrow 1 / 2$ that guarantees that $\left\langle\mathbf{s}^{\prime}(\xi, t) \mathbf{s}^{\prime}(\xi, t)\right\rangle=1$, as it should be for smooth lines (we recall that for pure random walking line the quantity $\left\langle\mathbf{s}^{\prime}(\xi, t) \mathbf{s}^{\prime}(\xi, t)\right\rangle$ does not exist at all). For large separation $\left(\xi_{2}-\xi_{1} \gg \xi_{0}\right)$ exponents tends to $\delta_{\alpha \beta}\left(2 \sqrt{\pi} \xi_{0} / 6\right) \delta\left(\xi_{1}-\xi_{2}\right)$, and correlation between tangent vectors weakens, $\left\langle\mathbf{s}^{\prime}(\xi, t) \mathbf{s}^{\prime}(\xi, t)\right\rangle \rightarrow 0$. The second term in parenthesis of expression (12) appears due to closeness of the loops. It ensures that

$$
\int_{0}^{l} \int_{0}^{l} d \xi_{1} d \xi_{2}\left\langle\mathbf{s}^{\prime}\left(\xi_{1}, t\right) \mathbf{s}^{\prime}\left(\xi_{2}, t\right)\right\rangle=\left\langle(\mathbf{s}(l, t)-\mathbf{s}(0, t))^{2}\right\rangle=0
$$

as it should be for the closed lines (see explanations in ([12])). Thus the characteristic functional with function $N$ satisfies to necessary "semifractal" behavior of line and will be used further for evaluation of the rate reconnection coefficients. Here it is necessary to do two important comments. First one concerns the "semifractal" structure of the vortex loop. In principle, reconnections events occur due random walking behavior with non-zero probability of the meeting of any two elements. However, to evaluate the according rates on base of relations (4), (7), we need to know not only an average of $\delta\left(\mathbf{S}\left(\xi_{2}, \xi_{1}, t\right)\right)$ but also an average of the Jacobian. But the latter includes averaged derivatives (with respect two both label variable $\xi_{2}, \xi_{1}$ and time). It is known fact however that quantities like $\left\langle\left|\mathbf{s}_{\alpha}^{\prime}\left(\xi_{1}\right)\right|\right\rangle,\left\langle\mathbf{s}_{\alpha}^{\prime}\left(\xi_{1}\right) \mathbf{s}_{\beta}^{\prime}\left(\xi_{2}\right)\right\rangle$ etc. are ill-defined for pure random walking structure, For that reason we need to smooth away the curve on small scales. The second remark concerns the mean radius of curvature $\xi_{0}$. This quantity appears here as important parameter of theory of random walk. It is frequently called as "an elementary step" or persistence length. In fact the theory described by the Wiener distribution is failed for small scales less then $\xi_{0}$, therefore usually this value appears as a low cut-off of the whole approach.

\section{EVALUATION OF $A\left(l_{1}, l_{2}, l\right)$ AND $B\left(l, l_{1}, l_{2}\right)$.}

\section{A. evaluation of $B\left(l, l_{1}, l_{2}\right)$}

Let us come back to the rate coefficients. We start with the self-intersection processes. Positions of line elements $\mathbf{s}\left(\xi_{2}, t\right), \mathbf{s}\left(\xi_{1}, t\right)$ and relative vector $\mathbf{S}_{b}\left(\xi_{2}, \xi_{1}, t\right)$ are strongly fluctuating quantities having the Gaussian statistics. Due to the Wick theorem the average in integrand of (4) can be taken as a sum of all possible pairs of quantity $\mathbf{S}_{b}\left(\xi_{2}, t, \xi_{1}, t\right)$ and its derivatives. Because of uniformity in $\xi$ space, quantity $\mathbf{S}_{b}\left(\xi_{2}, \xi_{1}, t\right)$ depends on $\left|\xi_{2}-\xi_{1}\right|$, for that reason all averages of structure $\left\langle\left(\partial X / \partial \xi_{1}\right) \delta\left(X\left(\xi_{2}, \xi_{1}, t\right)\right)\right\rangle$ vanish, therefore only pairs separately from $\mathbf{S}_{b}\left(\xi_{2}, \xi_{1}, t\right)$ and from its derivatives survive. As a result the average of production is equal to production of averages and each of the factors can be evaluated separately

$$
\left\langle\left|\frac{\partial(X, Y, Z)}{\partial\left(\xi_{2}, \xi_{1}, t\right)}\right|_{\zeta=\zeta_{a}} \delta\left(\mathbf{S}_{b}\left(\xi_{2}, \xi_{1}, t\right)\right)\right\rangle=\left\langle\left|\frac{\partial(X, Y, Z)}{\partial\left(\xi_{2}, \xi_{1}, t\right)}\right|_{\zeta=\zeta_{a}}\right\rangle\left\langle\delta\left(\mathbf{S}_{b}\left(\xi_{2}, \xi_{1}, t\right)\right)\right\rangle .
$$


As mentioned, the use of the characteristics functional (9), (11) allows to calculate any averaged functional of configurations $\{\mathbf{s}(\xi, t)\}$. Let us show how to evaluate $\left\langle\delta \mathbf{S}_{s}\left(\xi_{2}, \xi_{1}, t\right)\right\rangle$. With use of the standard integral representation for $\delta$-function

$$
\delta(x)=\frac{1}{(2 \pi)} \int_{-\infty}^{\infty} e^{i x y} d y
$$

we rewrite $\left\langle\delta \mathbf{S}_{b}\left(\xi_{2}, t, \xi_{1}, t\right)\right\rangle$ as

$$
\begin{aligned}
\left\langle\delta \mathbf{S}_{b}\left(\xi_{2}, \xi_{1}, t\right)\right\rangle= & \frac{1}{(2 \pi)^{3}} \int\left\langle\exp \left[i \mathbf{y}\left(\mathbf{s}\left(\xi_{2}, t_{2}\right)-\mathbf{s}\left(\xi_{1}, t_{1}\right)\right)\right]\right\rangle d^{3} \mathbf{y}= \\
& \frac{1}{(2 \pi)^{3}} \int\left\langle\exp \left(i \int_{\xi_{1}}^{\xi_{2}} \mathbf{y s}^{\prime}(\xi, t) d \xi d t\right)\right\rangle d^{3} \mathbf{y} .
\end{aligned}
$$

Comparing (9) and (14) we conclude that the integrand in last term of (14) is just the characteristic functional $W(\{\mathbf{P}(\xi, t)\})$, taken at value of $\mathbf{P}(\xi, t)$

$$
\mathbf{P}(\xi)=-\mathbf{y} \theta\left(\xi-\xi_{1}\right) \theta\left(\xi_{2}-\xi\right)
$$

Here $\theta(\xi)$ is the unit step-wise function. Relation (15) implies that we choose in integrand of the characteristic functional only points lying in interval from $\xi_{1}$ to $\xi_{2}$ on the curve. Substituting this value $\mathbf{P}(\xi, t)$ expressed by $(15)$ in CF (11) with function $N^{\alpha \beta}\left(\xi_{1}-\xi_{2}\right)$ in form (12) we obtain

$$
\left\langle\delta \mathbf{S}_{b}\left(\xi_{2}, t, \xi_{1}, t\right)\right\rangle=\frac{1}{(2 \pi)^{3}} \int \exp \left[-\mathbf{y}^{2} \frac{2 \sqrt{\pi} \xi_{0}}{6}\left(\left(\xi_{2}-\xi_{1}\right)-\left(\xi_{2}-\xi_{1}\right)^{2} / l\right)\right] d^{3} \mathbf{y}=\left(\frac{3}{2 \pi^{3 / 4} \xi_{0}\left(\left(\xi_{2}-\xi_{1}\right)-\left(\xi_{2}-\xi_{1}\right)^{2} / l\right)}\right)^{3 / 2}
$$

Evaluation of absolute value of Jacobian in (13) we perform by use of $|J|=\sqrt{J^{2}}$. Furthermore it would be convenient for the sake of generalization to use vector velocity $\mathbf{V}_{l}=d \mathbf{s} / d t$, instead of calculation of it in explicit form, expressing velocity via the vortex filament configuration $\{\mathbf{s}(\xi)\}$. Calculation of $J^{2}$ can be fulfilled writing Jacobian in explicit form and subsequent applying the Wick theorem. Simple but tedious calculations leads to result that

$$
J^{2}=2\left\langle\mathbf{V}_{l x}^{2}\right\rangle\left\langle\left(\partial \mathbf{s}_{y} / \partial \xi_{1}\right)^{2}\right\rangle\left\langle\left(\partial \mathbf{s}_{z} / \partial \xi_{2}\right)^{2}\right\rangle+2\left\langle\mathbf{V}_{l x}^{2}\right\rangle\left\langle\left(\partial \mathbf{s}_{y} / \partial \xi_{2}\right)^{2}\right\rangle\left\langle\left(\partial \mathbf{s}_{z} / \partial \xi_{1}\right)^{2}\right\rangle+p . p .
$$

where p.p. all permutations with respect to $x, y, z$. Estimating $\left\langle\left(\partial \mathbf{s}_{y} / \partial \xi_{1}\right)^{2}\right\rangle$ and similar terms as $1 / 3$ we obtain that $|J|=\frac{2}{3}\left|\mathbf{V}_{l}\right|$. After use of the integration $\iint d \xi_{1} d \xi_{2} \delta\left(\xi_{2}-\xi_{1}-l_{1}\right)$ (see 4) we finally obtain

$$
B\left(l_{1}, l-l_{1}, l\right)=b_{s} * V_{l} \frac{l}{\left(\xi_{0}\left(l_{1}-l_{1}^{2} / l\right)\right)^{3 / 2}}
$$

where constant $b_{s}=\left(\sqrt{3} / 8 \pi^{9 / 4}\right) \approx 0.0164772$. We introduced in coefficient $B$ the additional factor $1 / 2$ in order to avoid the over-counting of the reconnection events, since decays $l \rightarrow l_{1}+l_{2}$ and $l \rightarrow l_{2}+l_{1}$ describe the same process, though the both enter into equations. Let us recall that the quantity $\xi_{0}$ is the mean radius of curvature. The numerical factor $b_{s} \approx 0.0164772$ is the result of particular approximation used in paper ([12]). If for instance we used pure Wiener distribution with the persistency length $\xi_{0}$ and disregard the closeness of line, we would have for coefficient $B\left(l_{1}, l-l_{1}, l\right)$ the expression

$$
B_{C}\left(l_{1}, l-l_{1}, l\right)=b_{s} * V_{l} \frac{l}{\left(\xi_{0} l_{1}\right)^{3 / 2}}
$$

where $b_{s} \approx 0.11$. This result is very similar to result obtained earlier in paper [11] from the qualitative consideration. Yet it is important also that in this way it is not clear how to relate the persistency length $\xi_{0}$ with the mean radius of curvature. 


\section{B. evaluation of $A\left(l_{1}, l_{2}, l\right)$}

Let us now evaluate quantity $A\left(l_{1}, l_{2}, l\right)$ defined by relation (7). We again (as for the previous case) evaluate average from Jacobian and $\delta$-function separately. Contribution from Jacobian coincides with the previous result $|J|=\frac{2}{3}\left|\mathbf{V}_{l}\right|$. The rest $\delta$-function part can be evaluated with the help of the CF obtained above. Unlike previous case we have to know two-loop distribution function. Since we omit interaction of loops (until the reconnection event occurs) the CF for two loops with lengths $l_{1}$ and $l_{2}$ is just production of the expressions of type (11)

$$
\begin{aligned}
W\left(\left\{\mathbf{P}_{1}(\xi)\right\},\left\{\mathbf{P}_{2}(\xi)\right\}\right)= & \exp \left\{-\int_{0}^{l_{1}} \int_{0}^{l_{1}} \mathbf{P}^{\alpha}\left(\xi_{1}\right) N_{1}^{\alpha \beta}\left(\xi_{1}-\xi_{2}\right) \mathbf{P}^{\beta}\left(\xi_{2}\right) d \xi_{1} d \xi_{2}\right\} \times \\
& \exp \left\{-\int_{0}^{l_{2}} \int_{0}^{l_{2}} \mathbf{P}^{\alpha}\left(\xi_{1}\right) N_{2}^{\alpha \beta}\left(\xi_{1}-\xi_{2}\right) \mathbf{P}^{\beta}\left(\xi_{2}\right) d \xi_{1} d \xi_{2}\right\} .
\end{aligned}
$$

Quantities $N_{1}^{\alpha \beta}\left(\xi_{1}-\xi_{2}\right)$ and $N_{2}^{\alpha \beta}\left(\xi_{1}-\xi_{2}\right)$ differ from each other only by lengths of loops $l_{1}$ and $l_{2}$, entering expressions for $N_{1}^{\alpha \beta}$. Further, by use of the standard integral representation for $\delta$-function we have

$$
\left\langle\delta \mathbf{S}_{f}\left(\xi_{2}, \xi_{1}, t\right)\right\rangle=\frac{1}{(2 \pi)^{3}} \int\left\langle\exp \left[i \mathbf{y}\left(\mathbf{s}_{2}\left(\xi_{2}, t_{2}\right)-\mathbf{s}_{1}\left(\xi_{1}, t_{1}\right)\right)\right]\right\rangle d^{3} \mathbf{y} .
$$

We stress again that the label variables $\xi_{2}$ and $\xi_{1}$ belongs two different loops. Let us introduce initial points $\mathbf{s}_{1}(0)$ and $\mathbf{s}_{2}(0)$ and rewrite (21) in the following form:

$$
\frac{1}{(2 \pi)^{3}} \int \exp \left[-i \mathbf{y}\left(\mathbf{s}_{2}(0)-\mathbf{s}_{1}(0)\right)\right]\left\langle\exp \left[i \mathbf{y}\left(\mathbf{s}_{2}\left(\xi_{2}\right)-\mathbf{s}_{2}(0)\right)\right] \exp \left[-i \mathbf{y}\left(\mathbf{s}_{1}\left(\xi_{2}\right)-\mathbf{s}_{1}(0)\right)\right]\right\rangle d^{3} \mathbf{y}
$$

Identifying further the "initial" positions $\mathbf{s}_{1}(0), \mathbf{s}_{2}(0)$ with quantities $\mathbf{R}_{1}, \mathbf{R}_{2}$ in formula (7) we rewrite it as

$$
\begin{aligned}
A\left(l_{1}, l_{2}, l\right)= & \frac{1}{\operatorname{VOLUME}} \frac{2}{3}\left|\mathbf{V}_{l}\right| \iint d \mathbf{R}_{1} d \mathbf{R}_{2} \iint d \xi_{1} d \xi_{2} \\
& \frac{1}{(2 \pi)^{3}} \int \exp \left[-i \mathbf{y}\left(\mathbf{R}_{\mathbf{2}}-\mathbf{R}_{\mathbf{1}}\right)\right]\left\langle\exp \left[i \mathbf{y}\left(\mathbf{s}_{2}\left(\xi_{2}\right)-\mathbf{s}_{2}(0)\right)\right] \exp \left[-i \mathbf{y}\left(\mathbf{s}_{1}\left(\xi_{2}\right)-\mathbf{s}_{1}(0)\right)\right]\right\rangle d^{3} \mathbf{y} .
\end{aligned}
$$

Let us introduce variables $\mathbf{R}_{1}-\mathbf{R}_{2},\left(\mathbf{R}_{1}+\mathbf{R}_{2}\right) / 2$. Integration over $\mathbf{R}_{2}-\mathbf{R}_{1}$ gives $\delta(\mathbf{y})$, integration over $\left(\mathbf{R}_{1}+\mathbf{R}_{2}\right) / 2$ gives the total volume of system. Further, integration over $\mathbf{y}$ gives unity, and integration over $\xi_{1}, \xi_{2}$ gives the production $l_{1} l_{2}$. Thus we obtain the remarkable result, that for noninteracting loops quantity $A\left(l_{1}, l_{2}, l\right)$ responsible for merging of loops does not depend on statistics of individual loop at all and is equal

$$
A\left(l_{1}, l_{2}, l\right)=b_{m} V_{l} l_{1} l_{2}
$$

Here $b_{m}=1 / 3$. As earlier we introduced additional factor $1 / 2$ to avoid the over-counting of the reconnection events.

Results (23) and (18) (with not well determined factors $b_{s}$ and $b_{m}$ ) were also obtained in paper [11]. Authors used some qualitative picture of moving and colliding elements of lines. This fact confirms the validity of approach made in our work, which allows to use it for more complicated (in comparison with random walk) cases.

\section{CONCLUSION}

In the paper we discussed a role of kinetics of the merging and breaking down vortex loops in the whole vortex tangle dynamic. Following the work [11] we introduced the reconnection rates $A\left(l_{1}, l_{2}, l\right)$ and $B\left(l, l_{1}, l_{2}\right)$. Quantity $A$ is a number (per unit of time and per unit of volume) of the fusion of two loops of lengths $l_{1}$ and $l_{2}$ with formation of the single loop of length $l=l_{1}+l_{2}$. Quantity $B\left(l, l_{1}, l_{2}\right)$ describes rate of the breakdown of the loop with the length $l$ into two daughter loops with lengths $l_{1}$ and $l_{2}$. We developed mathematical formalism, which enables us to 
calculate $A\left(l_{1}, l_{2}, l\right)$ and $B\left(l, l_{1}, l_{2}\right)$. Briefly, these quantities can be found as an average number of zeroes of vector $\mathbf{S}_{b}\left(\xi_{2}, \xi_{1}, t\right)$ connecting points of $\xi_{2}$ and $\xi_{1}$ at moment of time $t$. The averaging procedure should be fulfilled by using statistics of individual loops, expressed e.g. by the probability distribution functional $\mathcal{P}(\{\mathbf{s}(\xi, t)\})$. We applied this formalism to evaluate the reconnection rates $A\left(l_{1}, l_{2}, l\right)$ and $B\left(l, l_{1}, l_{2}\right)$ in case when vortex loop is semifractal object, which is smooth for small separations along the line and has a random work structure for distant (along line) points. Relations (7) and (4)are the key results of the paper. They are formal, and do not depend on statistic of loop. They can be applied for any physical situation provided that we are able to get statistics from the dynamical problem. In that sense the application of the results (7) and (4) for Gaussian case is just illustration how to use it in concrete sense. One of serious problem in this illustration is that in Gaussian approach the low scale was restricted by mean radius of curvature. In real vortex tangle $\xi_{0}$ in superfluid turbulent HeII this scale exceeds greatly the scales at which the real dissipative mechanisms occur. Thus, the study of what would happen for scales smaller of mean radius of curvature is on of actual problems. Nevertheless results expressed by relations (23) and (18) are significant, they allows to analyze the kinetic equation (1), the attempts were made in papers [11], [13]. We also will apply these result in the forthcoming paper for study of the kinetic equation.

I am grateful to participants of the workshop "Superfluidity under Rotation" (Manchester, 2005) for useful discussion of the results exposed above. This work was partially supported by grant N 03-02-16179 of the Russian Foundation of Fundamental Research.

[1] R.J.Donnelly, Quantized Vortices in HeliumII, (Cambridge University Press, 1991).

[2] R.P.Feynman, in Progress in Low Temperature Physics, edited by C.J.Gorter (North-Holland, Ameterdam, 1955), Vol.I, p.17.

[3] W.F.Vinen, Proc. R. Soc. London A 242, 493(1957).

[4] S.K.Nemirovskii and W.Fiszdon, Rev. Mod. Phys. 67, 37 (1995).

[5] J.Koplik and H.Levine, Phys. Rev. Lett. 71, 1375 (1993).

[6] K.W.Schwarz, Phys. Rev. B 38, 2398 (1988).

[7] C.F.Barenghi, D.C.Samuels, G.H.Bauer and R.J.Donnelly, Phys. Fluids 9, 2631(1997).

[8] R.G.K.M.Aarts and A.T.A.M.de Waele, Phys. Rev. B 50, 10069 (1994).

[9] M. Tsubota, T. Araki and S. K. Nemirovskii, Phys. Rev. B 62, 11751 (2000).

[10] C.F.Barenghi and D.C.Samuels, Journal of Low Temperature Physics, Vol. 136, Nos. 5/6, (2004).

[11] E.J.Copeland, T.W.B.Kibble and D.A.Steer, Phys.Rev. D, textbf\{58, \} 043508, (1998).

[12] S.K. Nemirovskii, Phys. Rev. B 57, 5792 (1997).

[13] Joao Magueijoa, Havard Sandvik, and Dani‘ele A. Steer, http://lanl.arxiv.org/abs/astro-ph/9905363. 